\title{
ACHIKO-D350: A dataset for early AMD detection and drusen segmentation
}

\author{
Huiying Liu ${ }^{1}$, Yanwu Xu ${ }^{1}$, Damon W. K. Wong ${ }^{1}$, Augustinus Laude ${ }^{2}$, \\ Tock Han $\mathrm{Lim}^{2}$, and Jiang Liu ${ }^{1}$ \\ ${ }^{1}$ Institute for Infocomm Research, Agency for Science, Technology and Research, Singapore \\ ${ }^{2}$ National Healthcare Group Eye Institute, Tan Tock Seng Hospital, Singapore
}

\begin{abstract}
Age related macular degeneration is the third leading cause of global blindness. Its prevalence is increasing in these years for the coming of "aging population". Early detection and grading can prevent it from becoming severe and protect vision. Drusen is an important indicator for AMD. Thus automatic drusen detection and segmentation has attracted much research attention in the past years. However, a barrier handicapping the research of drusen segmentation is the lack of a public dataset and test platform. To address this issue, in this paper, we publish a dataset, named ACHIKO-D350, with manually marked drusen boundary. ACHIKO-D350 includes 254 healthy fundus images and 96 fundus images with drusen. The images with drusen cover a wide range of types, including images with sparsely distributed drusen or clumped drusen, images of poor quality, and both well macular centered images and mis-centered images. ACHIKO-D350 will be used for performance evaluation of drusen segmentation methods. It will facilitate an objective evaluation and comparison.
\end{abstract}

\section{Introduction}

Automatic early Age-related Macular Degeneration (AMD) detection and grading is important for both clinicians and patients. AMD, after cataract and glaucoma, is the third leading cause of blindness worldwide and the first leading cause in the elderly [7] [6]. At its early stage, usually referred to as early AMD, it has not any obvious symptoms therefore is usually unnoticed by patients. While the symptoms, e.g., vision scotoma and distortion et al., appear and noticed by the patient, it is usually too late to restore the affected vision. Regular screening is a potential strategy to detect AMD at the early stage. However, manual detection and grading of AMD is time-consuming and labor-intensive. More importantly, it is subjective and thus can cause inaccuracy. Therefore, automatic early AMD detection and grading has attracted much research effort in these years.

The major sign of early AMD is the appearance of drusen, which are the deposit of waste around macular appearing as yellow-white spots on digital fundus images, as shown in Fig. 1 (b) and (c). Drusen detection has significant importance for early AMD detection and drusen segmentation is important for AMD grading because the number, size, and type of drusen are important criteria for AMD grading. Here we refer to drusen detection as judging the existence of drusen in a fundus image without the 


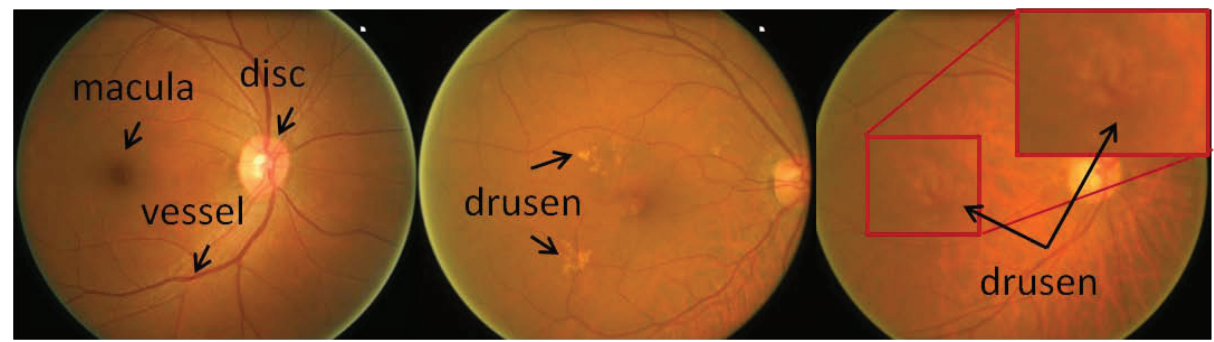

(a) Healthy retina

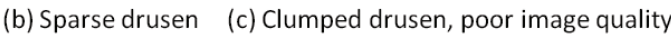

Fig. 1. Examples of fundus images with and without drusen. (a) A healthy fundus image. (b) A fundus image with sparse drusen. (c) A fundus image with clumped drusen, of poor image quality.

exact location and boundary, while drusen segmentation as finding the location and boundary of drusen.

The major difficulties of drusen detection and segmentation are:

1. Drusen do not have rigid shape or size (Fig. 1 (b) and (c)).

2. Drusen may appear differently due to different imaging conditions (Fig. 1 (c)).

3. The healthy structures of the fundus may be confused with drusen. For example, the vessels are usually dark and the pixels near vessels are often mistaken as drusen (Fig. 1 (c)).

4. In severe conditions the drusen may clump together thus increasing the difficulty of drusen segmentation (Fig. 1 (c)).

To address these difficulties, in recent years, lots of methods have been proposed for drusen segmentation and AMD detection. The earliest drusen segmentaion methods are based on local maxima, e.g., the geodesic method [2]. These methods first detect local maxima, then further classify the candidates according to contrast, size and shape. The second category consists of the local threshold based methods, e.g., Histogram based Adaptive Local Thresholding (HALT) [9], and Otsu method based adaptive threshold [10]. The third category includes the ones from frequency domain, e.g., wavelet [3], Fourier transform [12], and amplitude-modulation frequency modulation (AM-FM) [1].

Recently, some methods have been proposed to directly detect drusen-containing images without localizing drusen. These methods extract local features and then describe the whole image with these local features. The image is then taken as a whole and fed into a classifier and it is finally classified as containing drusen or not. The representative methods in this category include the method using biologically inspired features [4], and the method based on Hierarchical Word Image representation and SPIN features [14].

Although the methods detecting AMD with drusen segmentation show some progress, drusen segmentation has its importance in AMD grading. However, in spite of the existing dursen segmentation and detection methods, till now, there is not a publicly available test dataset and comparison platform. To facilitate the research of drusen segmentation, we extend the dataset Thalia [14], which is a dataset for early AMD detection, to include the boundary of drusen. The new dataset, named as ACHIKO-D350, is one of 

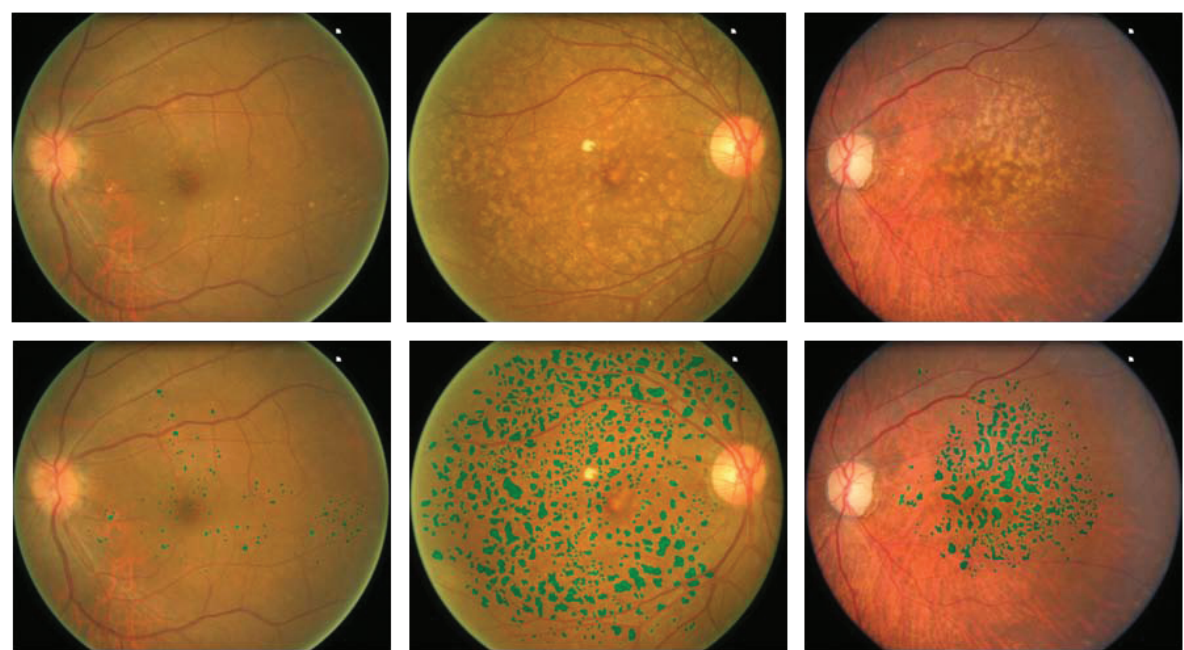

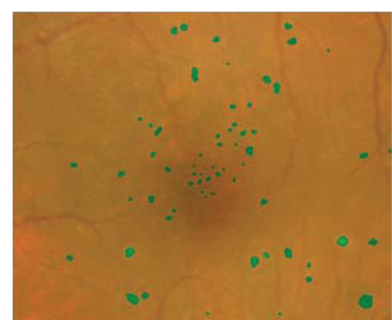

(a) Sparse \& small drusen

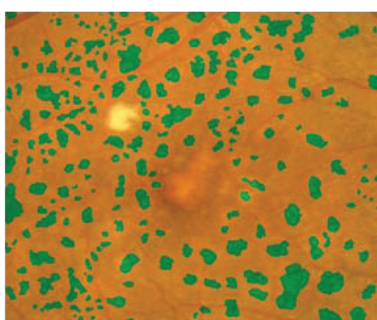

(b) Clumped \& many drusen

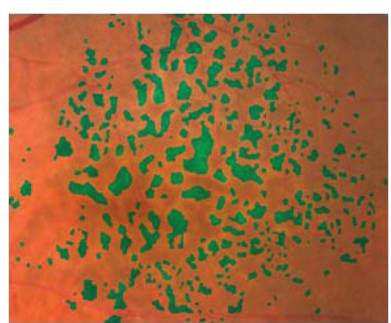

(c) Clumped \& many drusen

Fig. 2. Examples of sparse or clumped drusen. The first row shows the fundus image. The second row shows the fundus image with the drusen marked as green. The third row shows part of the marked fundus images enlarged.

the ACHIKO dataset series developped by iMED research team [8]. The images of the dataset are clinically diagnosed as AMD or non-AMD. For the AMD images, the boundary of drusen are semi-manually marked.

The rest of this paper is organized as follows. In the next section, we will state the details of the dataset, including the method and progress of data labeling, and the analysis of the dataset. In Section 3, we will compare our dataset with the existing dataset for early AMD detection. We will conclude this paper in Section 4.

\section{The dataset}

\subsection{Data source}

ACHIKO-D350 consists of 350 population-based images from the Singapore Eye Malay Study, consisting of 96 clinically verified drusen images and 254 non-drusen images. Each image has a resolution of $3072 \times 2048$ and had been acquired using a 45 FOV 

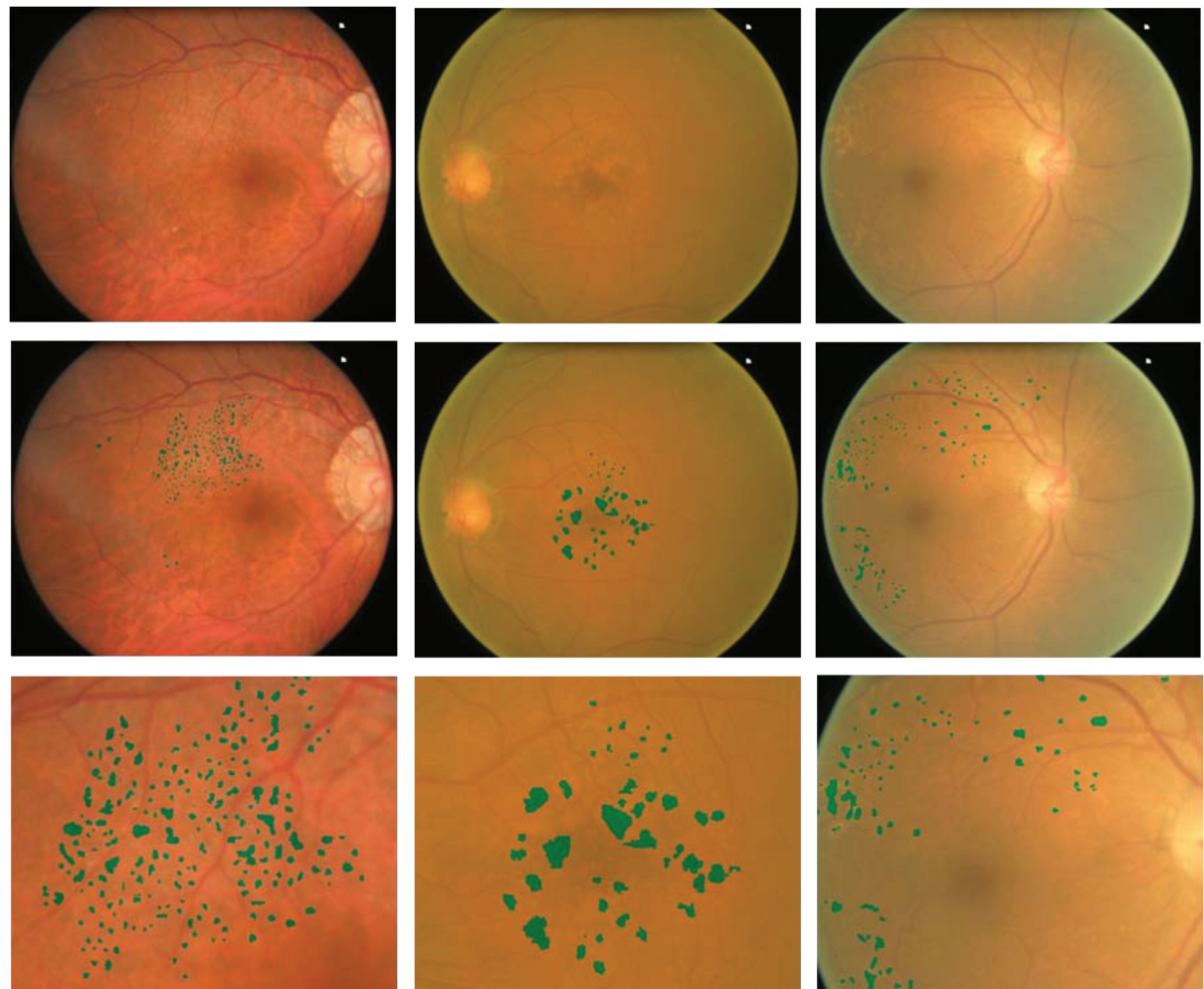

(a) Poor image quality

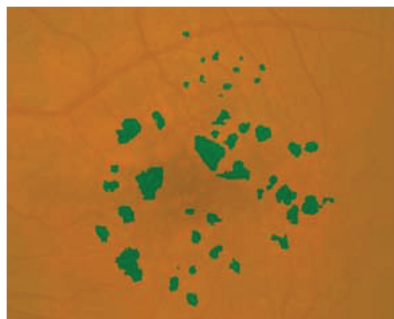

(b) Poor image quality

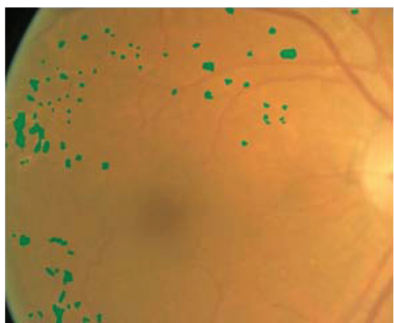

(c) Mis-centered image

Fig. 3. Examples of poor image quality and mis-centered image. The first row shows the fundus image. The second row shows the fundus image with the drusen marked as green. The third row shows part of the marked fundus images enlarged.

Canon CR-DGi retinal fundus camera with a 10D SLR backing. Out of the 96 images, 45 ones are of left eyes and 51 ones are of right eyes. Examples of the dataset are shown in the first row of Fig. 2 and Fig. 3.

\subsection{Drusen marking}

Manually marking the boundary is a tedious and labour intensive work. Therefore, we developed a semi-automatic labeling tool which is able to reduce the burden of labelling significantly. The graphic user interface of the tool is shown in Fig. 4. What the labeler need to do is to roughly mark drusen and non-drusen, by drawing rectangle, circle, free lasso, or other shapes. While marking, to avoid the merging of different drusen, the boundary between two drusen are marked as non-drusen. The user can choose from the full image a region of interest to be enlarged and do the marking on the enlarged figure. The already labeled drusen are shown as green in the figure. These designs make the labelling easier so more accurate. 


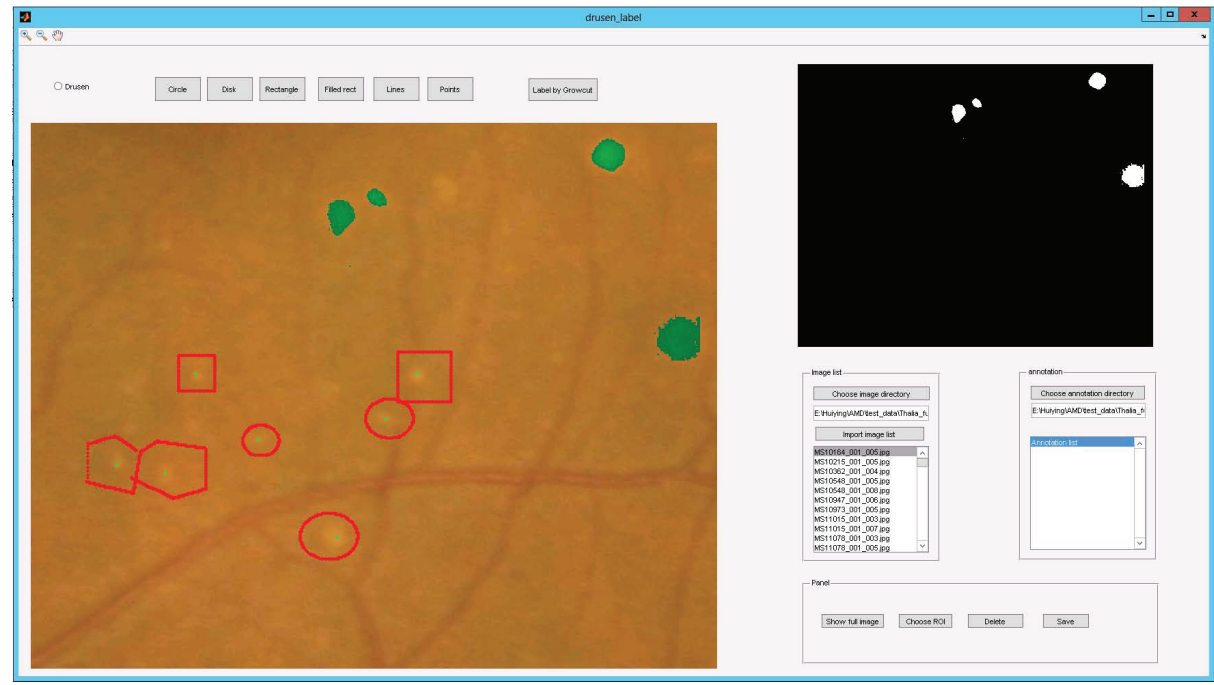

Fig. 4. The user interface of the marking tool. A part of the full image is enlarged and shown at the left of the figure. The regions in green are already labeled drusen. The green dots are marked by the labeler as drusen. The red rectangles/circles/lassos are marked as non-drusen. Growcut will use these information to get the boundaries of the drusen. The generated mask is shown at the right-top of the figure.

After roughly marking the drusen and the boundary, the labelling tool automatically tracks the exact boundary of the drusen. The Growcut segmentation method is employed for this task [13]. Growcut is a cellular automata based image segmentation method, verified to be effective in medical image segmentation. While labelling, we found that if the whole image is labelled at one time, Growcut is not able to give out satisfying result. Therefore, the local part covering the manual markers are fed into Growcut. If the boundary obtained are not satisfying, more manual marks will be added to improve the result.

Besides the boundary of drusen, the position of macula is also manually marked. Although there are already many methods for macula detection, e.g. macula detection method using seed mode tracking [15], it may fail under the situation of severe and dense drusen or poor image quality (Fig. 2, (c)).

The labelling work is done by a researcher with ocular image analysis background and then checked and verified by an ophthalmologist.

\subsection{Analysis of the dataset}

The dataset is diverse enough to cover many situations.

For AMD screening, macula centered fundus images are more useful than optic disc centered ones. But occasionally, the images are not well centered. Therefore, we need also mis-centered images. In the dataset, 14 ones are mis-centered images (examples 


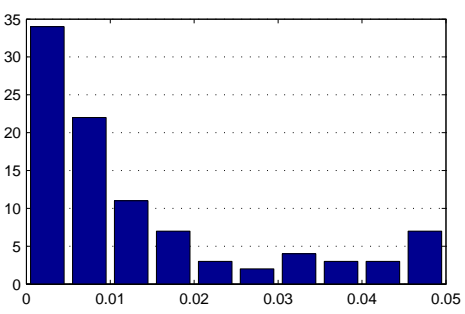

(a) histogram of drusen area

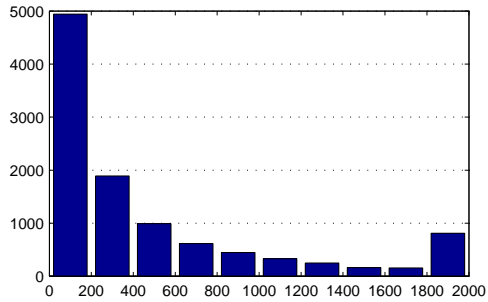

(c) histogram of drusen size

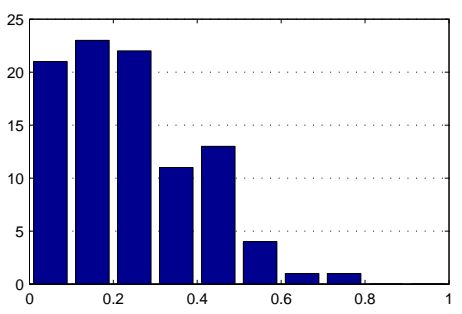

(b) histogram of drusen density

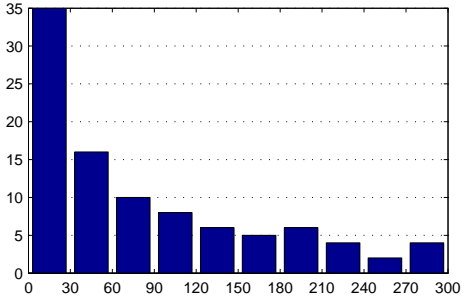

(d) histogram of drusen num

Fig. 5. Diversity analysis of the dataset. In each figure, the last bin is a "cut-off" bin. For example, in (a), the last bin covers all the samples larger than 0.045 .

can be found in Fig. 3 (c)) and the other 82 images are well centered ones (exampels can be found in Fig. 2, (a)-(c) and Fig. 3, (c)).

The quality of fundus image is affected by several factors, such as the size of pupil, cataract, and others. The poor quality of the fundus image may increase the difficulty of drusen segmentation. The dataset includes 14 fundus image of poor quality. Two examples are shown in Fig. 3 (a) and (b).

The dataset includes fundus images with various drusen size, maximum drusen density, number of drusen, and total drusen area. While calculating these properties, the image is resized to $1024 * 683$. While calculating drusen density, a window of the same size with disc $(70 * 70)$ is used. Fig. 5 shows the histogram of total drusen area, maximum drusen density, drusen size, and drusen number. From the figure, we can see that the dataset is diverse enough.

\section{Comparison with other datasets}

Currently, there are already several datasets for drusen detection but none of them include the boundary of drusen. These datasets are summarized in the following.

1. STARE, STructured Analysis of the REtina, is a dataset containing images of multiple diseases [3]. It contains 397 diginal fundus photos in total and ground truth is in the form of severity grades for the disease. The images are of resolution $700 \times 605$. Out of all the images, 62 were labeled as containing drusen, including 20 ones as large many, 13 ones as large few, 10 as fine many, and 19 as fine few. To the best 
of our knowledge, it is the first dataset containing drusen labeling. STARE also contains DR related lesions. 91 images are labeled as being affected by DR. It also contains manually labeling of vessels of part of the images.

2. ARIA, Automated Retinal Image Analysis, was published by St Pauls Eye Unit of Royal Liverpool University Hospital Trust in UK. It contains 212 images in total, including 92 ones with AMD, 61 normal ones, and 59 ones with diabetic retinopathy.

3. AREDS, Age-Related Eye Disease Study, enrolled 4,757 participants, aged 55-80 years. Among them, 3640 participants had at least early AMD and the other 1117 ones did not [5].

4. Thalia is a dataset constructed by iMED group from $I^{2} R$ (Institute of Infocomm Research, Singapore). It consists of 350 images, with 96 labeled as early AMD (drusen) and the others non-AMD (no drusen). Image resolution is $3072 \times 2048$ [14].

5. EUGENDA, EUropean GENetic DAtabase, is an ongoing project currently targets on AMD. Now it contains more than 4000 images with more than 191 ones containing drusen (http://www.eugenda.org/).

6. CAPT, Complications of Age-Related Macular Degeneration Prevention Trial, is a randomized clinical trial to evaluate whether prophylactic laser treatment to the retina can prevent the complications of the advanced stage of AMD. In total, 1052 patients with two high-risk eyes were enrolled. The images collected by CAPT can be used as dataset for automatic AMD detection [11].

The comparison of our dataset with the above mentioned ones are summarized in Table 1. Till now, ACHIKO-D350 is the only one with drusen boundary.

Table 1. Comparison with other datasets

\begin{tabular}{|l|l|l|l|l|l|}
\hline Dataset & \# AMD & \# non-AMD & total & $\begin{array}{l}\text { Drusen } \\
\text { Boundary }\end{array}$ & $\begin{array}{l}\text { AMD } \\
\text { grading }\end{array}$ \\
\hline STARE & 62 & 335 & 397 & No & Rough \\
ARIA & 92 & 120 & 212 & No & No \\
AREDS* & 1117 & 3640 & 4757 & No & No \\
EUGENDA** & $191+$ & & $4000+$ & No & No \\
CAPT* & 1052 & & & No \\
THALIA & 96 & 254 & 350 & No & No \\
ACHIKO-D350 & 96 & 254 & 350 & Yes & No \\
\hline
\end{tabular}

*The number is the number of patients, instead of number of images.

**This is an ongoing project. The number of images is increasing.

\section{Conclusion}

In this paper, we present a dataset, named ACHIKO-D350, for early AMD detection and drusen segmentation. As far as we know, this is the first publicly available dataset with 
marked drusen boundary. The dataset includes in total 350 images, with 254 heathy ones and 96 ones clinically diagnosed as AMD. The dataset covers diverse image conditions, e.g., poor image quality, sparse or clumped drusen. The direct usage of this dataset is performance evaluation of drusen detection and segmentation. Further potential usages lies in AMD grading.

\section{References}

1. E. Barriga, V. Murray, C. Agurto, M. Pattichis, S. Russell, M. Abramoff, H. Davis, and P. Soliz. Multi-scale am-fm for lesion phenotyping on age-related macular degeneration. In CBMS, pages 1-5, 2009.

2. Z. Ben Sbeh, L. D. Cohen, G. Mimoun, and G. Coscas. A new approach of geodesic reconstruction for drusen segmentation in eye fundus images. IEEE Transactions on Medical Imaging, 20(12):1321-1333, 2001.

3. L. Brandon and A. Hoover. Drusen detection in a retinal image using multi-level analysis. In MICCAI, pages 618-625. Springer, 2003.

4. J. Cheng, D. W. K. Wong, X. Cheng, J. Liu, N. M. Tan, M. Bhargava, C. M. G. Cheung, and T. Y. Wong. Early age-related macular degeneration detection by focal biologically inspired feature. In ICIP, pages 2805-2808, 2012.

5. M. Davis, R. Gangnon, L. Lee, L. Hubbard, B. Klein, R. Klein, F. Ferris, S. Bressler, R. Milton, et al. The age-related eye disease study severity scale for age-related macular degeneration: Areds report no. 17. Archives of ophthalmology, 123(11):1484, 2005.

6. R. D. Jager, W. F. Mieler, and J. W. Miller. Age-related macular degeneration. New England Journal of Medicine, 358(24):2606-2617, 2008.

7. R. Kawasaki, M. Yasuda, S. J. Song, S.-J. Chen, J. B. Jonas, J. J. Wang, P. Mitchell, and T. Y. Wong. The prevalence of age-related macular degeneration in asians: a systematic review and meta-analysis. Ophthalmology, 117(5):921-927, 2010.

8. J. Liu, D. Wong, Z. Zhang, B.-H. Lee, X. Gao, F. Yin, J. Zhang, and M. T. Htoo. Integrating research, clinical practice and translation: The singapore experience. In EMBC, pages 7148 $7151,2013$.

9. K. Rapantzikos, M. Zervakis, and K. Balas. Detection and segmentation of drusen deposits on human retina: Potential in the diagnosis of age-related macular degeneration. Medical image analysis, 7(1):95-108, 2003.

10. R. T. Smith, J. K. Chan, T. Nagasaki, U. F. Ahmad, I. Barbazetto, J. Sparrow, M. Figueroa, and J. Merriam. Automated detection of macular drusen using geometric background leveling and threshold selection. Archives of ophthalmology, 123(2):200, 2005.

11. C. study group. The complications of age-related macu- lar degeneration prevention trial (capt): rationale, design and methodology. pages 91-107, 2004.

12. K. Ujjwal, A. Chakravarty, and J. Sivaswamy. Visual saliency based bright lesion detection and discrimination in retinal images. In ISBI, pages 1428-1431, 2013.

13. V. Vezhnevets and V. Konouchine. Growcut: Interactive multi-label nd image segmentation by cellular automata. In Proc. of Graphicon, pages 150-156, 2005.

14. D. W. Wong, J. Liu, X. Cheng, J. Zhang, F. Yin, M. Bhargava, G. C. Cheung, and T. Y. Wong. Thalia-an automatic hierarchical analysis system to detect drusen lesion images for amd assessment. In ISBI, pages 884-887, 2013.

15. D. W. Wong, J. Liu, N.-M. Tan, F. Yin, X. Cheng, C.-Y. Cheng, G. C. Cheung, and T. Y. Wong. Automatic detection of the macula in retinal fundus images using seeded mode tracking approach. In $E M B C$, pages 4950-4953, 2012. 